\title{
Concept
}

\section{Sustainably Sourced Olive Polyphenols and Omega-3 Marine Lipids: A Synergy Fostering Public Health}

\author{
Mario Pagliaro, ${ }^{a}$ Daniela Maria Pizzone, ${ }^{b}$ Antonino Scurria,${ }^{a}$ Claudia Lino, ${ }^{a}$ \\ Emilia Paone, ${ }^{c}$ Francesco Mauriello, ${ }^{b *}$ Rosaria Ciriminna ${ }^{a *}$ \\ ${ }^{a}$ Istituto per lo Studio dei Materiali Nanostrutturati, CNR, via U. La Malfa 153, 90146 \\ Palermo, Italy \\ ${ }^{b}$ Dipartimento DICEAM, Università degli Studi Mediterranea di Reggio Calabria, Loc. Feo di \\ Vito, 89122 Reggio Calabria, Italy \\ ${ }^{c}$ Dipartimento di Ingegneria Industriale (DIEF), Università degli Studi di Firenze, via di Santa \\ Marta 3, 50139 Firenze, Italy \\ Thanks to the pioneering studies of Østerud and co-workers it is now increasingly understood \\ that natural polyphenols present in marine oils play an essential role in protecting omega-3 \\ lipids from oxidation and autooxidation ensuring that no proinflammatory products are formed \\ after intake as it often happens with assumption of refined omega-3 concentrates. Strong \\ antioxidants exerting multiple biological functions, olive biophenols are ideally suited to \\ functionalize marine oils creating a synergy which has the potential to foster public health \\ across the world. This study identifies suitable avenues to advance the sustainable production \\ of health-beneficial formulations based on newly obtained natural marine oils and olive \\ phenolic extracts. Important educational outcomes for bioeconomy educators conclude the \\ study.
}

\section{Introduction}

The numerous health benefits of the biophenols found in Olea europea include antiatherogenic, cardioprotective, anticancer, and neuroprotective activity [1]. Research in the field is flourishing with book chapters [2] and review articles [3] regularly summarizing newly discovered findings concerning the physiological role of these phenolic compounds, which include oleuropein, hydroxytyrosol, tyrosol and oleocanthal.

Being powerful antioxidants (free radical scavengers [4]), olive phenolic extracts can be used as highly effective non-toxic replacement for toxic, synthetic antioxidants used as preservative food additives [5]. These compounds show also significant anti-inflammatory activity. In the case of hydroxytyrosol (3,4-dihydroxyphenylethanol, HT) quantum mechanical calculations and molecular docking simulations suggest that the anti-inflammatory activity may be due to binding and inhibition of the lipoxygenase and cyclooxygenase enzymes [6].

Another key advantage of relevance in sight of pharmaceutical applications of these phenolics is their lack of toxicity. For example, HT is not genotoxic or mutagenic and its consumption is safe even at high doses [7].

Several olive phenolic extracts have been commercialized as nutraceutical formulations with multiple health benefits [3], ranging from prevention of cardiovascular disease to bone reinforcement, with the use of said formulations being at times supported by clinical evidence from randomized control trials [8].

Similarly, docosahexaenoic acid (DHA, C22:6n-3) and eicosapentaenoic acid (EPA, C20:5n-3) omega-3 (or $n-3$ ) long chain polyunsaturated fatty acids (PUFA) abundant in oily blue fish rich exhibit anti-inflammatory, neuroprotective and antithrombotic properties lowering heart rate and blood pressure [9], making consumption of fish and of blue fish in particular of fundamental relevance for physical and mental health of adults and children.

A new index (EPA+DHA in red blood cells) directly related to changes in EPA+DHA intake was introduced by Harris [10] in response to the main deficiency in diets common to industrially 
developed countries, namely the insufficient consumption of EPA and DHA.

Increasing the amount of EPA+DHA essential fatty acids in the body requires to either increase consumption of blue fish and seafood, or omega-3 supplementation. Growing at over $10 \%$ (compound) annual rate, the \$2.6 EPA/DHA ingredient global market in 2018 is estimated to almost triple by 2026 [11], further increasing overfishing pressure on anchovy and sardine stocks since anchovy/sardine oils are the dominant raw materials used to produce food and dietary supplement products.

The main production process currently employed to obtain fish oil omega-3 concentrate includes anchovy/sardine cooking and pressing on board the shipping vessel, followed by extensive chemical refining at omega-3 ingredient manufacturing sites [12].

Recently, simple and effective methods have been developed to sustainably source both olive polyphenols from olive and fish processing waste. In the case of olive phenolics, the process starts from olive mill wastewater, and employs citric acid to stabilize and progressively hydrolyze vegetation waters [13]. Extended to modern two-phase olive mills, the process affords olive phenolics aqueous extracts of unprecedented titer in hydroxytyrosol [14].

In the case of anchovy fish oil, a new process starts from anchovy processing waste and uses citrus-derived $d$-limonene as extraction solvent in a closed loop process in which the biobased solvent is fully recycled affording an oil rich not only in EPA and DHA [15] but also in valued vitamin $\mathrm{D}_{3}$ [16]. When compared to the multi-step conventional fish oil extraction and purification process, the new biobased circular process provides significant technical, economic and environmental advantages [17].

In this study, we show how combining sustainably sourced olive phenolic and omega-3 marine oil extracts may synergistically foster public health while providing substantial benefits to manufacturers of omega- 3 and olive phenolics ingredients. Important educational outcomes for bioeconomy educators conclude the study.

\section{The case for synergy between olive biophenols and omega-3 lipids}

An immediate advantage of using olive polyphenols in combination with omega-3 lipids is the enhanced chemical stability to PUFA tryglycerides abundant in marine oils.

This was shown by Spain's scholars in 2008 reporting that hydroxytyrosol of $90-95 \%$ purity obtained by hydrothermal treatment of olive oil byproduct (olive mill wastewater, OMWW) added in 50 and $100 \mathrm{ppm}$ to cod liver oil (40\% of omega-3 lipids) was able to inhibit $\omega-3$ lipids oxidation [18]. The higher oxidation inhibition was reached using 100 ppm HT (Figure 1).

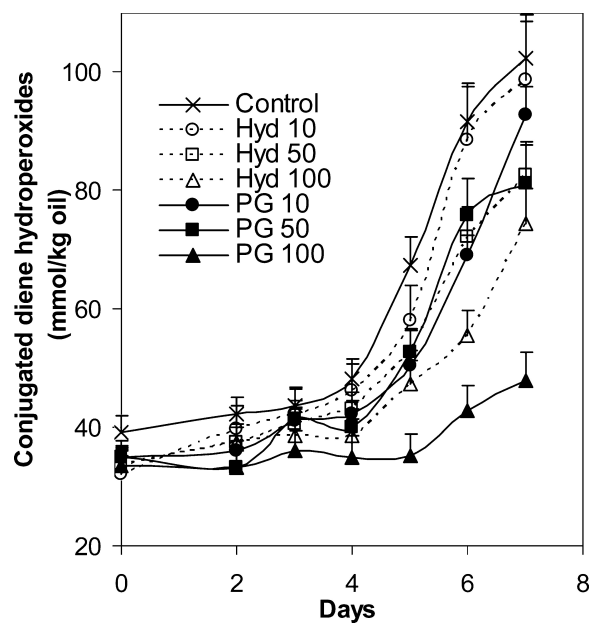

Fig. 1 Effect of hydroxytyrosol (Hyd) and propyl gallate (PG) at different concentrations $(10,50$, and $100 \mathrm{ppm})$ on the formation of conjugated diene hydroperoxides in fish oil during oxidation at $40{ }^{\circ} \mathrm{C}$. [Reproduced from Ref.18, with kind permission].

Exerting neuroprotective, anti-inflammatory, anticancer and even antiviral (against several influenza A viruses [19]) activities, hydroxytyrosol abundant in Olea europea was recently found to be "the most actively investigated natural phenol" [20].

The compound has limited antibacterial activity [21], but its great pharmacological potential is widely recognized [22]. For example, in late 2020, Italy's scholars reported the human safety of an oral spray containing microencapsulated hydroxytyrosol developed with the aim to inhibit the severe acute respiratory syndrome coronavirus 2 (SARS-CoV-2) endocytosis [23].

Showing evidence of industrial interest, a few months later a joint team including researchers from a large food company reported that OMWW is an excellent antioxidants to retard lipid oxidation in fish oil-enriched food products [24].

Noting how the antioxidant properties of OMWW in fish bulk oil had not been previously reported, the team showed how it is enough to add lyophilized OMWW in $1 \mathrm{wt} \%$ amount to tuna oil (5 wt\% in medium-chain triglycerides oil) to inhibit the formation of hydroperoxides, hexanal, and t,t-2,4-heptadienal throughout the storage period (14 days) by $94.2,96.5$, and $100 \%$, respectively. Volatile compounds hexanal and nonanal (oxidation products of $n-6$ PUFAs) and $t, t$-2,4-heptadienal (oxidation product of $n-3$ PUFAs) form during the storage of tuna oil, with hexanal being the main volatile formed during the storage. 
Table 1 Percent Inhibition of the Formation of Oxidation Compounds at the End of the Storage Study (14 days) [Adapted from Ref.24, with kind permission].

\begin{tabular}{lccc}
\hline Sample & $\begin{array}{c}\text { \% inhibition } \\
\text { peroxide }^{\text {values }}{ }^{\mathbf{a}}\end{array}$ & $\begin{array}{c}\text { \% inhibition } \\
\text { hexanal/hexanal- } \\
\mathbf{d}_{\mathbf{1 2}}\end{array}$ & $\begin{array}{c}\text { \% inhibition } \\
\text { heptadienal/hexanal-d }\end{array}$ \\
\hline Control & NA & NA & NA \\
EDTA & 75.5 & 51.0 & 71.7 \\
Oregano & 63.0 & 93.1 & 100 \\
OMWW & 94.2 & 96.5 & 100 \\
Parsley & 78.4 & 75.0 & 90.8 \\
Trolox & 68.3 & 35.2 & 63.7 \\
\hline
\end{tabular}

${ }^{\mathrm{a}} \mathrm{NA}$, not applicable

${ }^{b} \mathrm{POV}$ (peroxide values) expressed as mequiv $\mathrm{O}_{2} / \mathrm{kg}$ oil.

${ }^{\mathrm{c}}$ Ratio of peak areas. Hexanal- $\mathrm{d}_{12}$ was internal standard.

When compared to several antioxidants including oregano and parsley, ethylenediaminetetraacetic acid (EDTA) and Trolox (a water-soluble $\alpha$-tocopherol analogue), OMWW was found to be the best antioxidant (Table 1). Remarkably, the effectiveness of OMWW (as well as of parsley and oregano) as antioxidant was found to relate to the total phenol content and oxygen radical absorbance capacity (ORAC) but not to the iron(II) chelating activity.

In a complementary and straightforward approach, a marine oil rich in omega-3 lipids such as algae oil (AO) is directly mixed with extra virgin olive oil (EVOO) [25].

In detail, mixing a commercial AO with high concentration of $n$-3 PUFAs (35\% DHA, 20\% EPA and 5\% docosapentaenoic acid (DPA, C22:5n-3)) with a shelf-life of 1 month with EVOO with a minimum quantity of $60 \mathrm{mg} / \mathrm{g}$ of secoiridoids and $80 \%$ of oleic acid reduced the oxidation processes in a dosedependent manner (Figure 2) throughout storage.

Eventually, after 10 weeks, the amount of $n-3$ PUFAs in the commercial oil was reduced by about $30 \%$. It was enough to mix the AO oil with EVOO in 50:50 ratio (Mixture III) to lower the reduction in PUFA amount to $20 \%$. Eventually, in EVOO:AO 83:17 mixture the PUFA content after 10 weeks was reduced by less than $10 \%$.

The team concluded that the addition of EVOO rich in polyphenols to marine oils is "a simple alternative for the stabilization of unstable oils rich in omega-3, facilitating their application in the industry" [25].

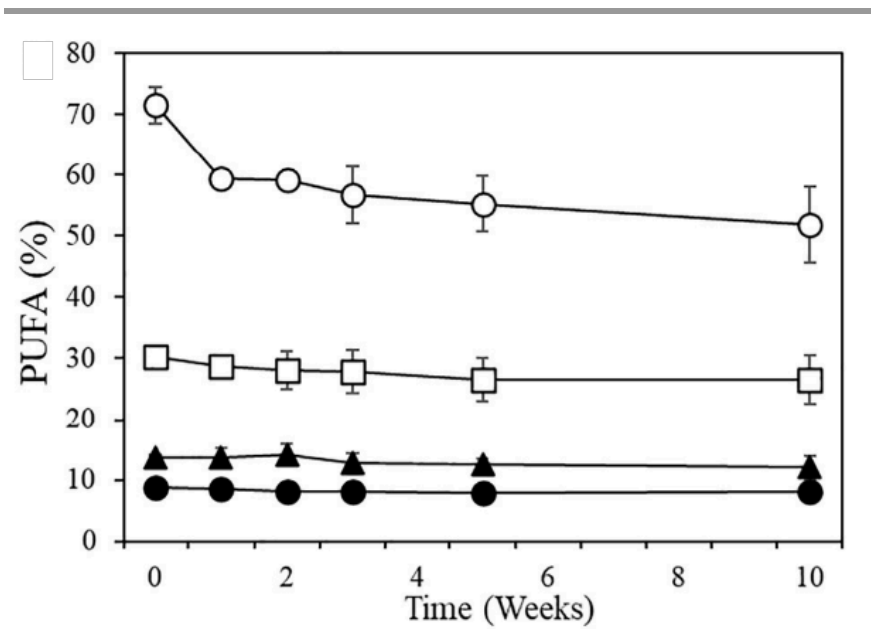

Fig. 2 Polyunsaturated Fatty Acids (PUFA; EPA+ DHA + DPA) concentration changes during accelerated storage conditions of EVOO, AO and mixtures. (a) (EVOO); (•) (Mixture I, AO:EVOO, 17:83 w/w); ( $\Delta$ ) (Mixture II, AO:EVOO, 25:75 $\mathrm{w} / \mathrm{w}$ ); ( $\square$ ) (Mixture III, AO:EVOO, 50:50 w/w); (O) (AO). Each value is represented as the mean as the mean $\pm S D ; n=3$ samples/oil. [Reproduced from Ref.25, with kind permission].

As mentioned above, synergy between olive polyphenols may extend well beyond the enhancement of omega-3 lipids oxidative stability.

For instance, as early as 2005 scholars in Brazil reported the outcomes of a clinical study showing that supplementation with olive oil in patients who had rheumatoid arthritis and were at the same time using fish oil supplements resulted in a more precocious and accentuated improvement when fish oil supplements were used in combination with olive oil [26].

The conventional industrial processes used to extract and purify fish oil [12] removes also the lipophilic polyphenols naturally present in the fish fat, including the powerful antioxidant and anti-inflammatory phlorotannins obtained by fish eating brown algae [27].

In 1986, studying the effect of eight weeks of daily intake of omega-3 lipids in the form of fish oil or omega-3 capsules, Østerud had found modest benefits on the activity of blood cells, suggesting that concentrated omega-3 did not have the same health effects as marine oils in natural form.

These preliminary findings were subsequently confirmed by numerous studies $[28,29,30]$, suggesting that a daily fish intake more effectively enhances the plasma concentration of $n-3$ PUFAs as compared with the intake of concentrated EPA and DHA ethyl esters in capsules.

Østerud and Elvevoll in Norway in the late 1990s first suggested that purification significantly lowers the health benefits of refined marine oils, freed of important natural antioxidants. In 2001 they reported at a congress held in Vienna the results of administering healthy volunteers with cold pressed versus refined marine oils. Better results, a consistent 
improvement on parameters related to the development of cardiovascular disease were noted by supplementation with cold pressed seal oil, despite a lower content of $n-3$ fatty acids in the unrefined oil [29].

Three years before, studying the effect of intake of various marine oils on the number and volume of platelets in 266 healthy volunteers, the team reported that the combination of cod liver oil and olive oil produces better effects than the oils given separately [31].

A few years later, they were the first to recognize the commercial relevance of olive polyphenols to fish oil-based supplements applying for a patent describing the combination of seal oil and cold-pressed virgin olive oil [32]. The new combined oil was dubbed "Olivita".

A clinical study was carried out during which healthy subjects were given $15 \mathrm{~mL}$ /day of combined cold pressed olive oil with refined seal or fish oil for 10-14 weeks. The recombined oils regained the properties lost during refining with clear antiinflammatory effects seen in reduction of MCP-1 (monocyte chomotactic protein-1), C-reactive protein (CRP), as well as thromboxane $\mathrm{B}_{2}$ and leukotriene $\mathrm{B}_{4}$ assuming the seal oil combined with olive oil, and reduction in cytokines when assuming the fish oil combined with olive oil [33]. In brief, the combined EVOO + marine oils behaved similarly to cold pressed marine oils, while said changes were not observed in fish and seal oils without added EVOO.

In light of these and previous findings, scholars today ascribe the absence of diabetes and coronary hearth disease in native Greenland Eskimos discovered by Bang and Dyerberg in the late 1960s and subsequently ascribed to high hematic levels of DHA and EPA abundant in seal and whale meat [34], also "at least partly... to their co-ingestion of phlorotannins" [35].

Indeed, the use of brown algal phlorotannins to prevent refined fish oil from rancidification was proposed as early as of 1996 when scholars in China reported that the antioxidant activity of $1 \%$ high molecular weight phlorotannins extracted from Sargassum kjellmanianum was 2.6 times higher than that of $0.02 \%$ BHT (tert-butyl-4-hydroxytoluene), a commonly employed synthetic antioxidant [36].

Today an omega-3 dietary supplement consisting of omega-3 from seal oil, extra virgin olive oil obtained from Spain and vitamin $\mathrm{D}$ is produced in Norway and successfully commercialized across the world. The manufacturer recommends the intake of six capsules per day (and not 1 or 2 as it happens with omega-3 capsules using refined marine oils) because the omega-3 fatty acids are not concentrated [37].

This suggests that newly developed olive biophenol extracts may reduce the number of needed capsules while enhancing the amount of phenolic compounds whose benefits, as mentioned above, go well beyond simple antioxidant activity.

\section{The case for concentrated olive biophenol extracts}

Most extraction routes of olive polyphenols from different sources such as olive leafs or olive mill wastewater afford extracts with phenolics concentrations generally $<20 \mathrm{wt} \%$, and often $<10 \mathrm{wt} \%$ [3]. A recently developed extraction and separation protocol for olive mill wastewater polyphenols, on the other hand, affords concentrated phenolic oils [38] ideally suited to be mixed with marine oils.

In detail, raw OMWW obtained from olive mills undergoes centrifugation and simple filtration to remove all solid particles after which it is acidified to $\mathrm{pH} \approx 2$ with concentrated $\mathrm{HCl}$, and defatted in a separatory funnel using $n$-hexane. The resulting aqueous layers are extracted with EtOAc to retrieve the phenolic molecules, after which the EtOAc extract is dried over anhydrous $\mathrm{Na}_{2} \mathrm{SO}_{4}$, and evaporated under vacuum affording a yellowish-brown crude oil.

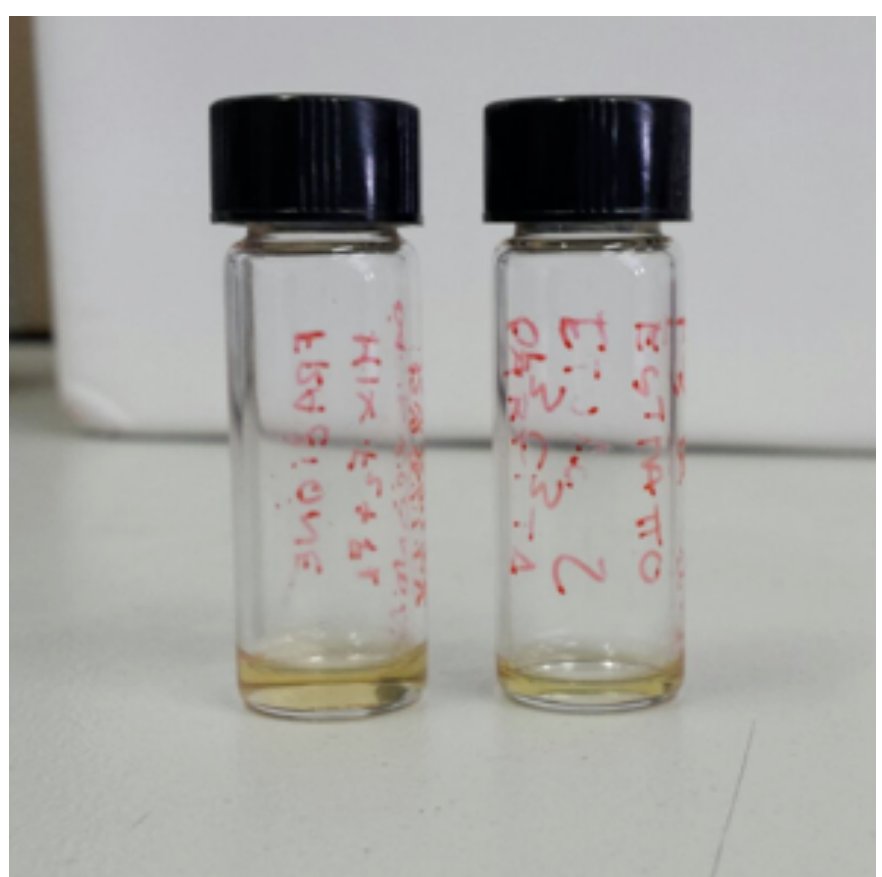

Fig. 3 Typical olive biophenol extracts obtained via liquid-liquid extraction of OMWW followed by chromatographic purification through a silica-based column. [Reproduced from Ref.41, Creative Commons Attribution 4.0 International License].

The crude extract is purified by dissolving it in EtOAc, followed by addition of powdered silica and solvent evaporation under reduced pressure. The oil adsorbed on silica is loaded onto a silica gel septum packed in $n$-hexane which is first eluted with hexane to remove any residual apolar component, and then with EtOAc to recover the biophenol fraction. The eluate is separated from the solvent via evaporation under reduced pressure affording a yellowish oil 
comprised of olive phenolics (silica purified polyphenol mixes, SPPM).

The same crude samples obtained from liquid-liquid extraction were purified also through a C18-reversed phase silica column packed in 9:1 acetonitrile:acidified water $(2.5 \% \mathrm{w} / \mathrm{w}$ formic acid). The column was eluted with the latter eluent and then polarity was gradually increased up to $1: 1$ acetonitrile: acidified water in order to recover a biophenol fraction obtained as a yellowish oil (C18 purified polyphenol mixes, CPPM). The oils (Figure 3) have a virtually identical phenolics profile, with tyrosol and hydroxytyrosol as the main components (Table 2).

Table 2 Amounts of hydroxytyrosol and tyrosol in the SPPM and CPPM extracts [Reproduced from Ref.39, with kind permission].

\begin{tabular}{lcc} 
Sample & Hidroxytyrosol $(\mathbf{m g} / \mathbf{L})$ & Tyrosol $(\mathbf{m g} / \mathbf{L})$ \\
\hline $\begin{array}{l}\text { Biancolilla } \\
\text { SPPM }\end{array}$ & $65.90 \pm 3.29$ & $16.04 \pm 0.81$ \\
$\begin{array}{l}\text { Biancolilla } \\
\text { CPPM }\end{array}$ & $63.34 \pm 3.16$ & $18.25 \pm 0.91$ \\
Cerasuola SPPM & $125.43 \pm 6.27$ & $29.85 \pm 1.49$ \\
$\begin{array}{l}\text { Cerasuola } \\
\text { CPPM }\end{array}$ & $127.81 \pm 6.37$ & \\
\hline
\end{tabular}

Aimed to avoid the use of harmful $n$-hexane, the industrial preparation of the similar phenolic extracts will employ biobased limonene [39] to defat the raw OMWW, and C18reversed phase silica as optimal stationary phase since it enables the use of water as a mobile phase combined with less polar solvents including environmentally friendly ethyl lactate [40].

OMWW is a virtually unlimited source of these valued compounds. It is enough to quickly assess the amount of hydroxytyrosol and tyrosol contained therein and then to apply the aforementioned green commercial extraction process to OMWW batches with the highest amount of these polyphenols [41].

Anchovy oil extracted with citrus-derived $d$-limonene from anchovy filleting waste affords a natural (non-refined) oil rich in EPA and DHA [15], vitamin $\mathrm{D}_{3}$ [16] and natural astaxanthin (Figure 4). Found in several microalgae, fish and seafood species, the latter carotenoid is a powerful antioxidant and an important neuroprotective agent [42].

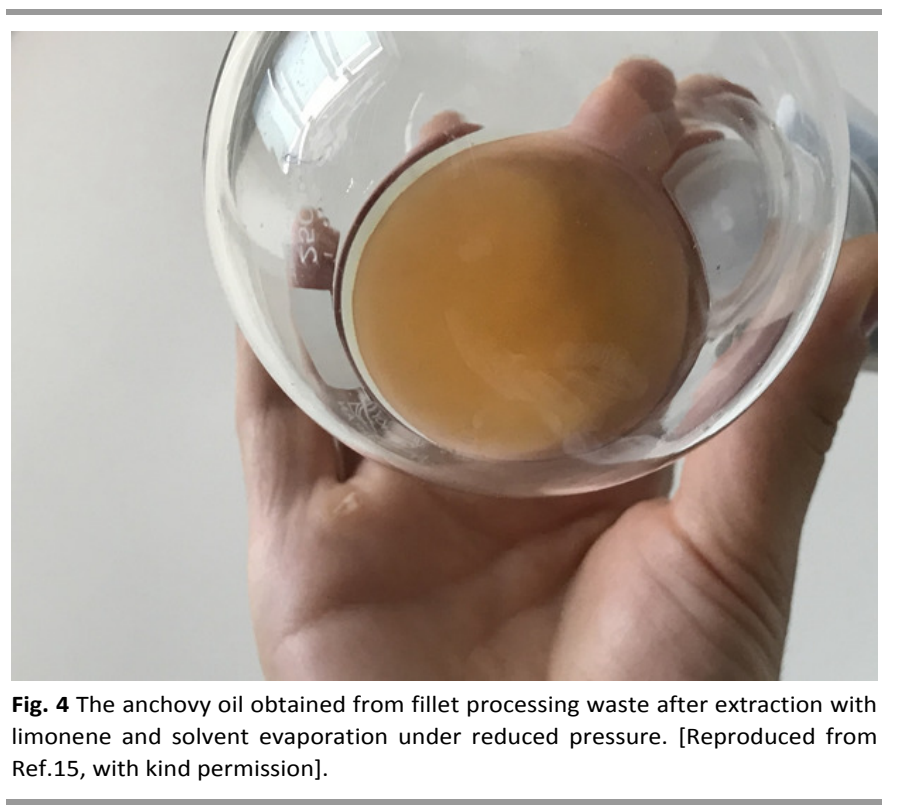

Even for this newly developed marine oil dubbed herein AnchoisOil, the addition of the new olive biophenol extracts described above would further protect the PUFA and astaxanthin from oxidation. At the same time, the intake of such a functionalized natural fish oil would results in cardoprotective action thanks to the olive biophenols integrated into the lipoproteins after entering the bloodstream protecting their lipid components from oxidation and exerting anti-inflammatory action at the artery walls [35].

Clinical studies will be necessary to demonstrate the synergy between this new marine oil and concentrated olive biophenols in the prevention of diseases such as coronarial hearth disease or in the cure (relieving) of common morbidities such as atherosclerosis, joint and muscle pain or psoriasis.

\section{Conclusions and perspectives}

Largely thanks to the pioneering studies of Østerud, it is now increasingly understood that supplementation of refined omega3 polyunsaturated lipids in the form of ethyl esters may cause either limited effect on human blood lipids, platelets and coagulation [43] or even undesirable oxidative stress leading to increase in proinflammatory products [44].

Østerud and Elevoll suggest that the lack of cardiovascular disease and type 2 diabetes observed in the late 1960s by Dyerbeg and Bang in native Greenlanders chiefly eating seal and whale blubber [34] has been due to the concomitant action of omega-3 lipids and the natural polyphenols contained in blubber protecting the unstable PUFAs from oxidation and autooxidation [45].

Aware of the unique antioxidant power of olive polyphenols [4], the Norway's scholars in the mid 1990s started to study the 
clinical effects of mixing unrefined marine oils with extra virgin olive oil rich in phenolic compounds [31].

Eventually, the combination of marine oils and EVOO was patented [32] and new nutraceutical products based protecting against oxidative degradation and provided with further antiinflammatory, anti-atherogenic and cardioprotective activities due to synergy between olive biophenols and omega-3 lipids were commercialized [37].

Subsequent advances in marine oil and olive biophenol extraction technologies have opened the route to the production of new nutraceutical and pharmacological products based on the combination of newly obtained natural marine oils and olive phenolics extracts.

In the case of marine oils, a new general strategy to obtain highly valued marine oils in their natural form, namely as tryglycerides containing plentiful natural antioxidants including astaxanthin, was demonstrated for both anchovy [15] and shrimp [46] waste (rather than from anchovy and shrimp) using biobased and biocompatible citrus limonene as recyclable extraction solvent.

In the case of olive phenolics, new green processes were demonstrated to readily obtain highly concentrated and pure phenolic extracts in two steps using liquid-liquid extraction followed by chromatography on hydrophobized silica $[39,41]$.

In the era of overfishing [47] and with the global population rapidly ageing $(22 \%$ of the world's projected population is expected to be over the age of 60 years by 2050 , from just $12 \%$ in 2015 [48]), these findings are of remarkable global relevance.

Rather than mixing olive and marine oils, the aforementioned natural marine oils obtained from overfished and mercury-free marine species, such as anchovy and shrimp processing waste will be further functionalized with small amounts of olive oil waste extracts rich in pluripotent biophenols such as hydroxytyrosol, tyrosol and oleocanthal.

Following clinical studies, the nutraceutical and pharmacological products based on the aforementioned combination of newly obtained natural marine oils and olive phenolics extracts will hopefully provide and expand the health benefits already observed via the combination of marine and olive oils.

New green chemistry technologies such as the green extraction and recovery of natural products summarized in this study for marine oils and olive phenolics actually behave as the key enabling technologies of the bioeconomy, with important educational consequences for green chemistry and bioeconomy educators using recent research achievements to foster student creativity [49].
Students indeed find in this case study another example of how also in biochemistry and in the bioeconomy progress in science is far from being linear, and requires the re-discovery of forgotten findings [50] such as those concerning the unique role of antioxidants in marine oils and the dangers associated with their removal during the production of refined fish oil to manufacture food and nutraceutical supplements $[12,43,44,45]$.

As the chemical industry rapidly evolves towards biobased productions carried out in distributed chemical plants using green chemistry technologies [51], furthermore, students can learn how the production of marine oils and phenolic extracts at low cost enabled by the aforementioned green chemistry routes allows to expand their commercial utilization of fishery and agricultural waste in low-income and middle-income countries, meeting a key requirement of the bioeconomy for which highly valued functional substances are sustainably derived from renewable resources [52].

\section{Author information}

\section{Corresponding Authors}

*E-mail: rosaria.ciriminna@cnr.it

*E-mail: francesco.mauriello@unirc.it

\section{ORCID}

Mario Pagliaro: 0000-0002-5096-329X

Daniela Maria Pizzone: 0000-0002-3909-3545

Antonino Scurria: 0000-0001-5624-6833

Claudia Lino: 0000-0002-8724-7772

Emilia Paone: 0000-0001-8184-750X

Rosaria Ciriminna: 0000-0001-6596-1572

Francesco Mauriello: 0000-0002-6002-2151

\section{Notes}

The research received no external funding. The Authors declare no conflict of interest.

\section{Acknowledgements}

This work is dedicated to Professor Bjarne Østerud, University of Tromsø, for all he has done to elucidate the role of natural antioxidants in marine oils.

\section{References}

1 A. Karković Marković, J. Torić, M. Barbarić, and C. Jakobušić Brala, Hydroxytyrosol, Tyrosol and Derivatives and Their Potential Effects on Human Health, Molecules 2019, 24, 2001.

2 D. Boskou, Phenolic Compounds in Olives and in Olive Oil, In Olive Oil: Minor Constituents and Health, D. Boskou (Ed.), CRC Press, Boca Raton (FL): 2009; pp.11-44. 
3 R. Ciriminna, A. Fidalgo, F. Meneguzzo, L. M. Ilharco, M. Pagliaro, Extraction, Benefits and Valorization of Olive Polyphenols, Eur. J. Lipid Sc. Technol. 2016, 118, 503-511.

4 F. Visioli, G. Bellomo, C. Galli, Free radical-scavenging properties of olive oil polyphenols, Biochem. Biophys. Res. Commun. 1998, $247,60-64$.

5 R. Ciriminna, F. Meneguzzo, R. Delisi, M. Pagliaro, Olive Biophenols as New Antioxidant Additives in Food and Beverage, ChemistrySelect 2017, 2, 1360-1365.

6 C. Semidalas, E. Semidalas, M. T. Matsoukas, C. Nixarlidis, P. Zoumpoulakis, In silico studies reveal the mechanisms behind the antioxidant and anti-inflammatory activities of hydroxytyrosol, Med. Chem. Res. 2016, 25, 2498-2511.

7 F. Echeverría, M. Ortiz, R. Valenzuela, L. A. Videla, Hydroxytyrosol and Cytoprotection: A Projection for Clinical Interventions, Int. J. Mol. Sci. 2017, 18, 930.

8 D. Trombetta, A. Smeriglio, S. Cascapera, C. Colica, An hydroxytyrosol-based pharmaceutical formulation for the prevention of cardiovascular disease: a randomized controlled crossover trial, Biomed. Prevent. 2017, 1, 62-69.

9 H. Zirpoli, C. L. Chang, Y. A. Carpentier, A. T. Michael-Titus, V. S. Ten, R. J. Deckelbaum, Novel Approaches for Omega-3 Fatty Acid Therapeutics: Chronic Versus Acute Administration to Protect Heart, Brain, and Spinal Cord, Annu. Rev. Nutr. 2020, 40, 161-187.

10 W. S. Harris, The Omega-6:Omega-3 ratio: A critical appraisal and possible successor, Prostag. Leukotr. Ess. 2018, 132, 34-40.

11 Global Market Insights, EPA/DHA (Omega 3) Ingredients Market Size By Source (Anchovy/Sardine Oil, High Concentrates, Medium Concentrates, Low Concentrates, Algae Oil, Tuna Oil, Cod Liver Oil, Salmon Oil, Krill Oil, Menhaden Oil), By Application (Dietary supplements, Pharmaceuticals, Functional Foods, Pet \& Animal Feed, Infant Formulas), Industry Analysis Report, Regional Outlook, Application Potential, Price Trends, Competitive Market Share \& Forecast, 2019 - 2026, Selbyville (DE): 2019.

12 R. Ciriminna, F. Meneguzzo, R. Delisi, M. Pagliaro, Enhancing and improving the extraction of omega-3 from fish oil, Sustain. Chem. Pharm. 2017, 5, 54-59.

13 R. Crea, Method of Obtaining a Hydroxytyrosol-rich Composition from Vegetation Water, US20020058078, 2001.

14 R. Delisi, R. Ciriminna, S. Arvati, F. Meneguzzo, M. Pagliaro, Olive Biophenol Integral Extraction at a Two-Phase Olive Mill, J. Clean. Prod. 2018, 174, 1487-1491.

15 R. Ciriminna, A. Scurria, G. Avellone, M. Pagliaro, A Circular Economy Approach to Fish Oil Extraction, ChemistrySelect 2019, 4, 5106-5109.

16 A. Scurria, C. Lino, R. Pitonzo, M. Pagliaro, G. Avellone, R. Ciriminna, Vitamin $\mathrm{D}_{3}$ in Fish Oil Extracted with Limonene from Anchovy Leftovers, Chem. Data Coll. 2020, 25, 100311.

17 R. Ciriminna, A. Scurria, A. S. Fabiano-Tixier, C. Lino, G. Avellone, F. Chemat, M. Pagliaro, Omega-3 Extraction from Anchovy Fillet Leftovers with Limonene: Chemical, Economic and Technical Aspects, ACS Omega 2019, 4, 15359-15363.

18 M. Pazos, A. Alonso, I. Sánchez, I. Medina, Hydroxytyrosol prevents oxidative deterioration in foodstuffs rich in fish lipids, J. Agr. Food Chem. 2008, 56, 3334-3340.
19 K. Yamada, H. Ogawa, A. Hara, Y. Yoshida, Y. Yonezawa, K. Karibe, V. B. Nghia, H. Yoshimura, Y. Yamamoto, M. Yamada, K. Nakamura, K. Imai, Mechanism of the antiviral effect of hydroxytyrosol on influenza virus appears to involve morphological change of the virus, Antiviral Res. 2009, 83, 35-44.

20 M. Bertelli, A. K. Kiani, S. Paolacci, E. Manara, D. Kurti, K. Dhuli, V. Bushati, J. Miertus, D. Pangallo, M. Baglivo, T. Beccari, S. Michelini, Hydroxytyrosol: A natural compound with promising pharmacological activities, J. Biotechnol. 2020, 309, 29-33.

21 M. S. Medina-Martínez, P. Truchado, P. Truchado, I. Castro, A. Allende, Antimicrobial activity of hydroxytyrosol: A current controversy, Biosci. Biotech. Biochem. 2015, 80, 1-10.

22 A. Karković Marković, J. Torić, M. Barbarić C. J. Brala, Hydroxytyrosol, Tyrosol and Derivatives and Their Potential Effects on Human Health, Molecules 2019, 24, 2001.

23 S. Paolacci, M. R. Ceccarini, M. Codini, E. Manara, S. Tezzele, M. Percio, N. Capodicasa, D. Kroni, M. Dundar, M. C. Ergoren, T. Sanlidag, T. Beccari, M. Bertelli, Pilot study for the evaluation of safety profile of a potential inhibitor of SARS-CoV-2 endocytosis (Endovir Stop), Acta Bio Med 2020, 91 (13-S):e2020009.

24 D. Jimenez-Alvarez, F. Giuffrida, P. A. Golay, C. Cotting, A. Lardeau, B. J. Keely, Antioxidant Activity of Oregano, Parsley, and Olive Mill Wastewaters in Bulk Oils and Oil-in-Water Emulsions Enriched in Fish Oil, J. Agric. Food Chem. 2008, 56, 16, 7151-7159.

25 D. González-Hedström, M. Granado, A. M. Inarejos-García, Protective effects of extra virgin olive oil against storage-induced omega 3 fatty acid oxidation of algae oil, NF J. 2020, 21, 9-15.

26 A. A. Berbert, C. Rosa Mitiko Kondo, C. Lisete Almendra, T. Matsuo, I. Dichi, Supplementation of fish oil and olive oil in patients with rheumatoid arthritis, Nutrition 2005, 21, 131-136.

27 M. Dutot, R. Fagon, M. Hemon, P. Rat, Antioxidant, antiinflammatory, and anti-senescence activities of a phlorotannin-rich natural extract from brown seaweed Ascophyllum nodosum, Appl. Biochem. Biotechnol. 2012, 167, 2234-2240.

28 F. Visioli, P. Rise, M. C. Barassi, F. Marangoni, C. Galli, Dietary Intake of Fish $v s$. Formulations Leads to HigherPlasma Concentrations of $n$-3 Fatty Acids, Lipids 2003, 38, 415-418.

29 E. O. Elvevoll, B. Østerud, Impact of processing on nutritional quality of marine food items, Forum Nutr. 2003, 56, 337-340.

30 E. O. Elvevoll, H. Barstad, E. S. Breimo, J. Brox, K.-E. Eilertsen, T. Lund, J. O. Olsen, B. Østerud, Enhanced Incorporation of n-3 Fatty Acidsfrom Fish Compared with Fish Oils, Lipids 2006, 41, 11091114.

31 E. Vognild, E. O. Elvevoll, J. Brox, R. L. Olsen, H. Barstad, M. Aursand, B. Østerud, Effects of dietary marine oils and olive oil on fatty acid composition, platelet membrane fluidity, platelet responses, and serum lipids in healthy humans, Lipids 1998, 33, 427-436.

32 E. O. Elvevoll, B. Østerud, Kombinasjon av oljer, samt anvendelse derav, NO20041197A, 2004.

33 B. Østerud, E. O. Olvevoll, The combination of virgin olive oils and refined marine oils. Beneficial effects, Progr. Nutr. 2008, 10, 230236.

34 J. Dyerberg, H. O. Bang, Haemostatic function and platelet polyunsaturated fatty acids in Eskimos, Lancet 1979, 2, 433-435. 
35 P. R. Clayton, S. Ladi, From alga to omega; have we reached peak (fish) oil?, J. R. Soc. Med. 2015, 108, 351-357.

36 X. J. Yan, X. C. Li, C. X. Zhou, X. Fan, Preservation of fish oil rancidity by phlorotannins from Sargassum kjelmaniamum, J. Appl. Phycol. 1996, 8, 201-203.

37 Olivita, Omega 3 Capsules, 2020. See at the URL: https://en.olivita.com/produkter/capsules/ (accessed: October 15, 2020).

38 R. Delisi, M. Pagliaro, F. Saiano, R. Ciriminna, C18 Alkyl-Modified Silica: A Suitable Tool for Olive Biophenol Green Extraction, Chem. Data Coll. 2017, 7-8, 102-106.

39 R. Ciriminna, M. Lomelli, P. Demma Carà, J. Lopez-Sanchez, M. Pagliaro, Limonene: A Versatile Chemical of the Bioeconomy, Chem. Commun. 2014, 50, 15288-15296.

40 F. Micăle, F. Albu1, E.-E. Iorgulescu, A. Medvedovici, F. Tache, Ethyl lactate as a greener alternative to acetonitrile in RPLC: a realistic appraisal, J. Chromatogr. Sci. 2015, 53, 1701-1707.

41 R. Delisi, F. Saiano, M. Pagliaro, R. Ciriminna, Quick assessment of the economic value of olive mill waste water, Chem. Cent. J. 2016, 10, 63-66.

42 C. Galasso, I. Orefice, P. Pellone, P. Cirino, R. Miele, A. Ianora, C. Brunet, C. Sansone, On the neuroprotective role of astaxanthin: new perspectives?, Mar. Drugs 2018, 16, 247.

43 D. W. T. Nilsen, K. Dalaker, A. Nordøy, B. Østerud, O. C. Ingebretsen, V. Lyngmo, S. Almdahl, J. Vaage, K. Rasmussen, Influence of a Concentrated Ethylester Compound of $n-3$ Fatty Acids on Lipids, Platelets and Coagulation in Patients Undergoing Coronary Bypass Surgery, Thromb. Haemost. 1991, 66, 195-201.

44 H. Arnesen, I. Seljeflot, Studies on very long chain marine $n-3$ fatty acids in patients with atherosclerotic heart disease with special focus on mechanisms, dosage and formulas of supplementation, Cell. Mol. Biol. 2010, 56, 18-27.

45 B. Østerud, E. O. Olvevoll, Dietary omega-3 fatty acids and risk of type 2 diabetes: lack of antioxidants?, Am. J. Clin. Nutr. 2011, 94 , 617-618.

46 A. Scurria, A.-S. Fabiano Tixier, C. Lino, M. Pagliaro, G. Avellone, F. D'Agostino, F. Chemat, R. Ciriminna, High Yields of Shrimp Oil Rich in Omega-3 and Natural Astaxanthin from Shrimp Waste, ACS Omega 2020, 5, 17500-17505.

47 J. S. Link, R. A. Watson, Global ecosystem overfishing: Clear delineation within real limits to production, Sci. $A d v . \mathbf{2 0 1 9}, 5$, eaav0474.

48 Opening the door to treating ageing as a disease, Lancet Diabetes Endocrinol. 2018, 6, 587.

49 M. Pagliaro, Chemistry education fostering creativity in the digital era, Isr. J. Chem. 2019, 59, 565-571.

50 J.-M. Lévy-Leblond, (Re)mettre la science en culture: de la crise épistémologique à l'exigence éthique, Seminar "Marcello Carapezza", Palermo, 27 March 2007. See at the URL: https://hal.archives-ouvertes.fr/hal-01197326/file/C56Levy.pdf

51 M. Pagliaro, An industry in transition: The chemical industry and the megatrends driving its forthcoming transformation, Angew. Chem. Int. Ed. 2019, 58, 11154-11159.

52 M. Pagliaro, The Central Role of Chemistry in the Transition to the Solar Economy: The Outcomes of two Lectures at the Russian Academy of Sciences, Gen. Chem. 2020, 6, 200007. 